\title{
Judicious utilisation of surgical and advanced radiotherapy resources to manage triple trouble: a case of synchronous dual malignancies with severe cervical spine deformity
}

\author{
Irfan Ahmad, ${ }^{1}$ Rahul Lohan, ${ }^{2}$ Kundan Singh Chufal, ${ }^{3}$ Sudip Raina ${ }^{4}$
}

'Department of Radiation Oncology, Batra Hospital and Medical Research Centre, New Delhi, Delhi, India

${ }^{2}$ Department of Diagnostic Radiology, Khoo Teck Puat Hospital, Yishun, Singapore ${ }^{3}$ Department of Radiation Oncology, Rajiv Gandhi Cancer Institute and Research Centre, New Delhi, Delhi, India ${ }^{4}$ Department of Surgical Oncology, Batra Hospital and Medical Research Centre, New Delhi, Delhi, India

Correspondence to Dr Irfan Ahmad, irfan.a@icloud.com

Accepted 1 April 2018
Check for updates

To cite: Ahmad I, Lohan $\mathrm{R}$ Chufal KS, et al. BMJ Case Rep Published Online First: [please include Day Month Year]. doi:10.1136/bcr-2018225096

\section{DESCRIPTION}

A 63-year-old man with a 20-year history of right torticollis and atlantoaxial rotational deformity (figure 1) presented with complaints of a non-healing painless ulcer over the right lateral aspect of tongue and difficulty swallowing for the preceding 3 months. On examination, an ulceroproliferative growth was noted on the right lateral border of tongue, involving the floor of mouth, outer alveolar border of mandible and crossing the midline without reaching the base of tongue. There was no palpable cervical lymphadenopathy

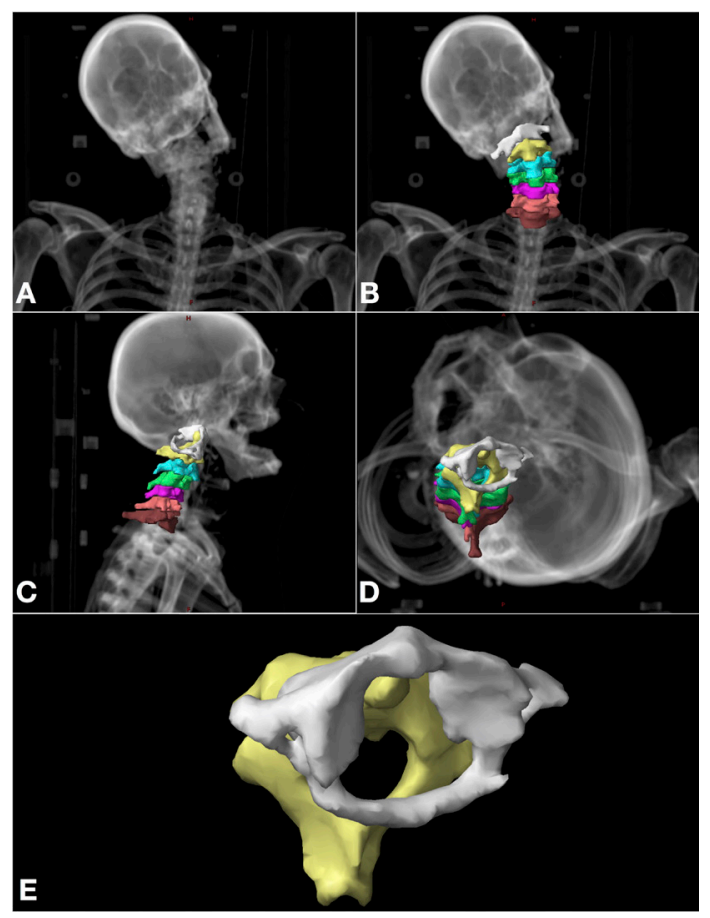

Figure 1 (A-D) Multiplanar 3D volume rendered, DRRs from the CT dataset, in AP, lateral and top-down views, demonstrate right lateral deviation of the cervical spine. Each cervical vertebra has been rendered in a separate colour to highlight the deformity (C1-white, C2-yellow, C3-cyan, C4-green, C5-purple, C6-orange, C7brown). (E) 3D top-down view of the first two cervical vertebras (atlas in white, axis in yellow) demonstrates rotatory subluxation at the atlantoaxial joint without anterior displacement (Fielding and Hawking Type 1 deformity). 3D, three dimensional; $A P$, anteroposterior; DRR, digitally reconstructed radiographs. bilaterally. An edge biopsy from the lesion revealed squamous cell carcinoma (SqCC) and a staging workup was initiated. Routine haematological investigations and chest X-ray were normal, however, a direct fibreoptic endoscopy revealed another exophytic growth in the left pyriform fossa (PFS) and a biopsy revealed SqCC. The

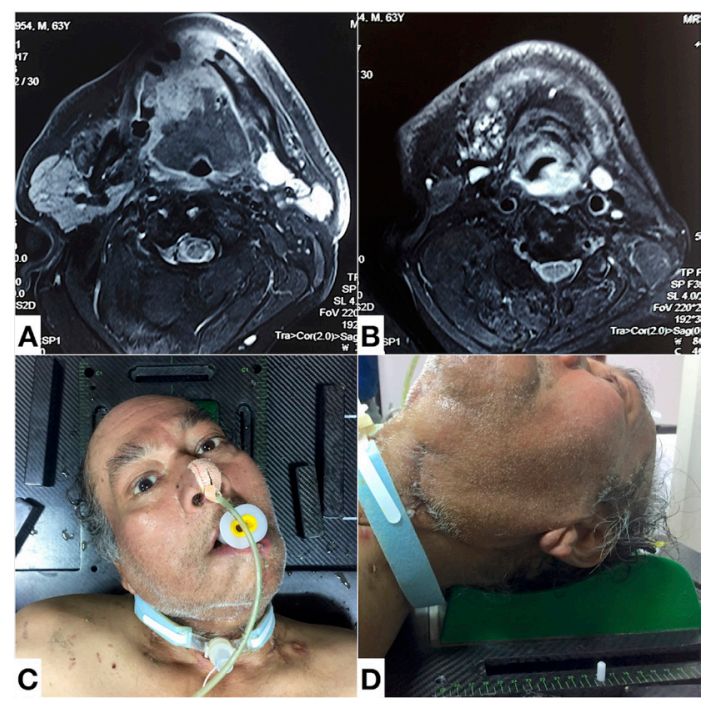

Figure $2 \mathrm{MRI}$ and patient positioning images. (A) Axial STIR MRI image demonstrates hyperintense nodular tumour at the floor of mouth on the right side with destruction of the adjacent mandible. (B) Another axial image from the same series shows the nodular hyperintense tumour in the left pyriform sinus. (C-D) Frontal and lateral images of the patient at the time of radiotherapy treatment positioning. Since the cervical spine was deviated in the right lateral direction, the patient was positioned with the intent to provide the most comfortable and reproducible treatment position. First, the neck rest was inverted to provide support to the head. An oropharyngeal airway was placed to provide separation between the tongue and hard palate, as well as to provide ready access in case suctioning of secretions had to performed. Finally, a custom perforated thermoplastic cast was made (not shown here) with openings for the nose, oropharyngeal airway and tracheostomy tube. The patient underwent a treatment planning CT of the face, neck and upper thorax and was simulated on Siemens Somatom Sensation Open (Siemens Healthineers, Erlangen, Germany) with a slice thickness of $2 \mathrm{~mm}$. STIR, short TI inversion recovery. 


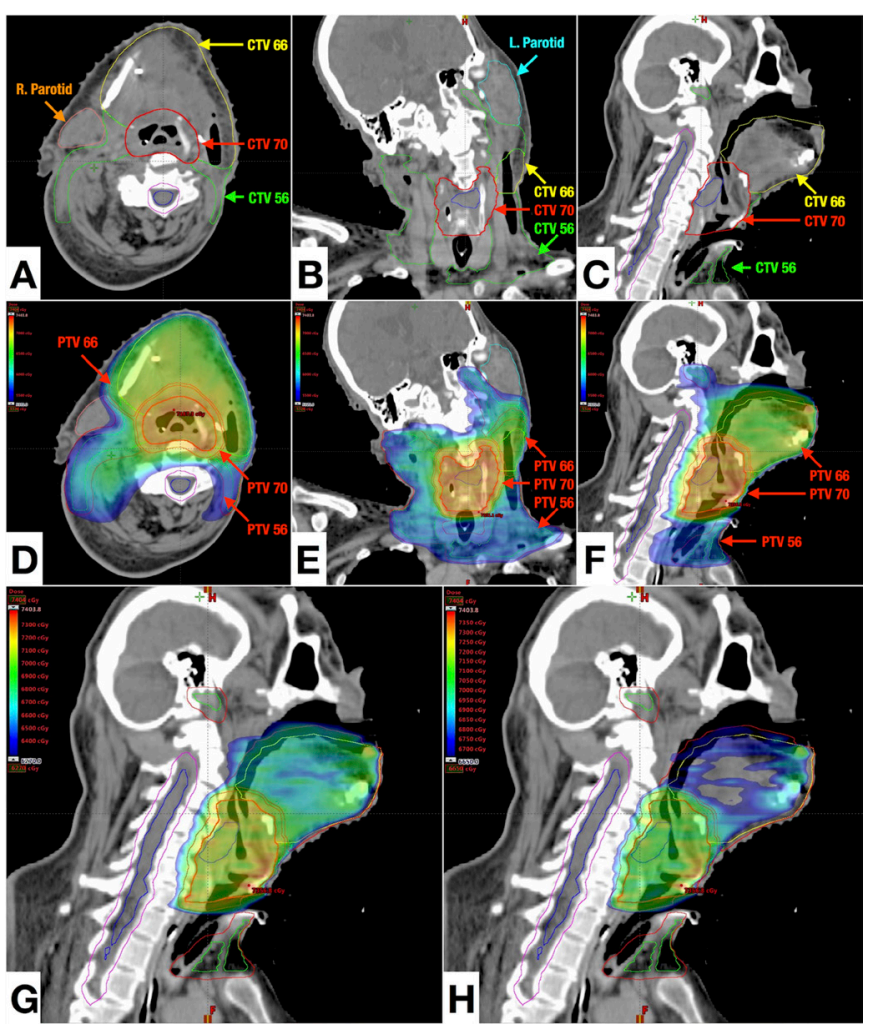

Figure 3 Treatment planning images. (A, B, C) Axial, coronal and sagittal sections of the patient showing CTVs and normal structures. CTV 70 (red contour) shows the volume receiving 70 Gy delineated for right PFS gross disease. CTV 66 (yellow contour) shows the volume receiving 66 Gy delineated for the operated bed of the tongue primary. Surgical clips can be seen in the axial section. CTV 56 (green contour) shows the elective nodal volume receiving $56 \mathrm{~Gy}$. Left and right parotid glands are also shown in cyan and orange contours, respectively. The spinal cord and its planning organ at risk volume are also shown in blue and purple contours, respectively. (D, E, F) Dose colour wash in axial, coronal and sagittal sections (with the lower limit set to $95 \%$ of $56 \mathrm{~Gy}$ ) depict adequate coverage of all PTVs (created by expanding the respective CTVs by $4 \mathrm{~mm}$, as per our institutional protocol) via simultaneous integrated boost technique. Treatment was delivered in a total of 35 fractions. $(G, H)$ Dose colour wash in the sagittal plane with the lower limit set to $95 \%$ of $66 \mathrm{~Gy}$ and $95 \%$ of $70 \mathrm{~Gy}$, depict adequate coverage of the respective PTVs and sparing of the spinal cord. CTV, clinical target volume; Gy, Gray; PFS, pyriform fossa; PTV, planning target volume.

patient underwent an MRI (face and neck) (figure 2a-b), and the case was discussed in our multidisciplinary tumour (MDT) board meeting. The patient was staged as synchronous dual primary of the head and neck, and each site was staged separately (tongue, $\mathrm{cT}_{4 \mathrm{a}} \mathrm{cN}_{0} \mathrm{cM}_{0}$, AJCC stage IVa; PFS, $\mathrm{cT}_{2} \mathrm{cN}_{0} \mathrm{cM}_{0}$, AJCC stage II). The specific issues discussed in the MDT were: (1) appropriate management of the dual primaries; (2) challenges posed by the spinal deformity on intraoperative anaesthesia and postoperative care and (3) difficulties in radiotherapy target delineation due to unusual anatomy.

The management options discussed in MDT were: (1) surgical resection for both lesions; (2) surgical resection of the tongue lesion and definitive radiotherapy to the PFS lesion or (3) definitive chemoradiation to both lesions. Surgical resection of both lesions was excluded due to the extensive nature of the required surgery, and definitive chemoradiation was excluded due to

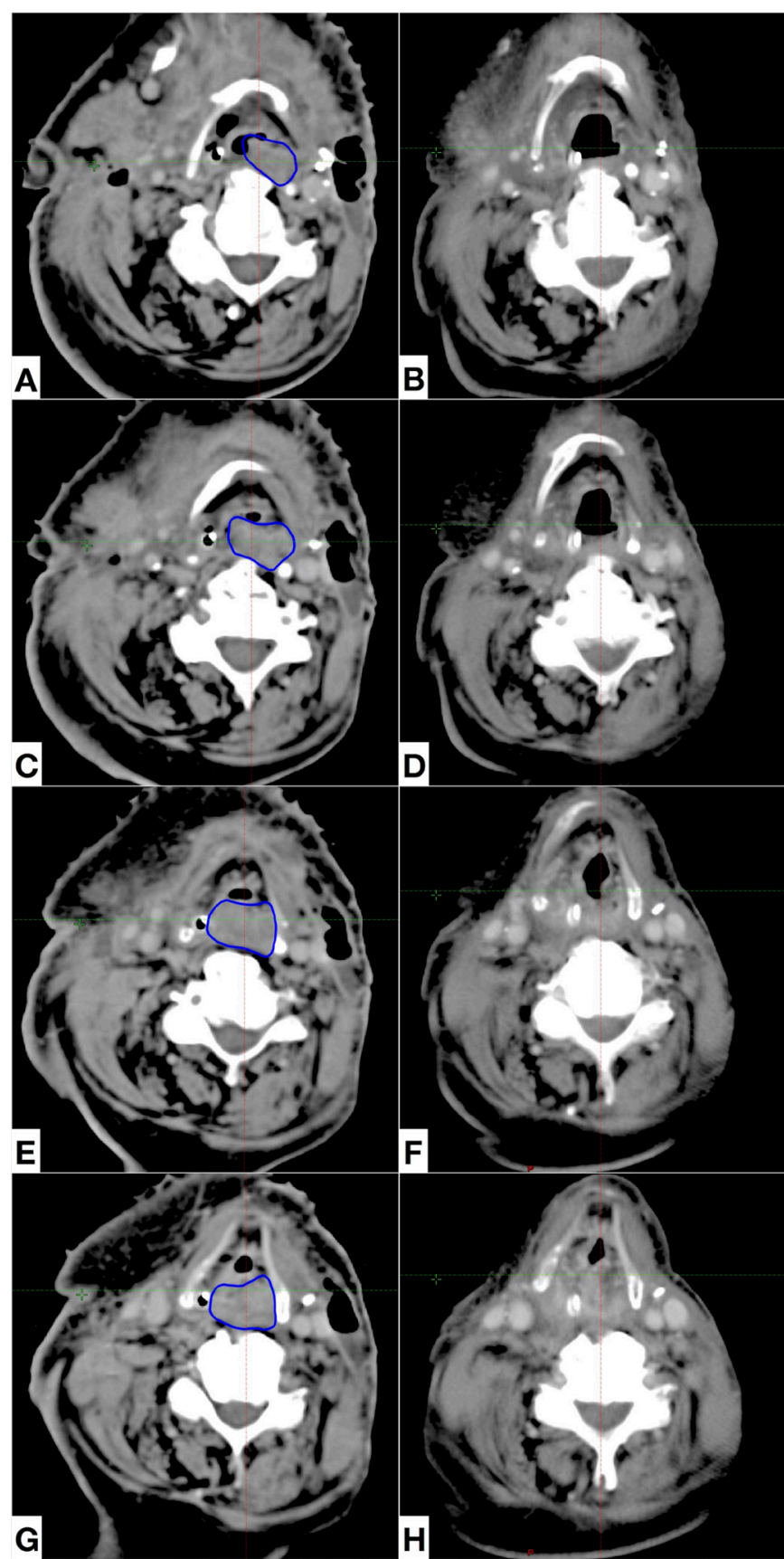

Figure 4 Paired axial pretreatment and post-treatment contrastenhanced CT images of the patient, with rigid registration between both datasets (after setting the region of interest as the larynx and hypopharynx) showing radiological complete response. Images on the left $(A, C, E, G)$ are from the pretreatment $C T$ and images on the right $(B, D, F, H)$ are from the post-treatment CT. Pretreatment images also show the gross tumour volume in the left PFS (blue contour). ( $A, B$ ) Axial sections at the upper border of hyoid bone. (C, D) Axial sections at the lower border of the hyoid bone. (E, F) Axial sections at the top of the thyroid cartilages. $(G, H)$ Axial sections at the level of midthyroid cartilages. PFS, pyriform fossa.

inferior local control rates in oral cavity SqCC. ${ }^{1}$ The patient underwent a hemiglossectomy, central arch resection and bilateral neck dissection along with an elective suprasternal tracheostomy (prior to the procedure) to facilitate airway management during surgery and the postoperative period. Histopathological evaluation revealed well-differentiated SqCC (depth of invasion 
Learning points

- Cervical spine deformities are challenging comorbidities to address in the context of modern oncological care and are unfortunately not captured in clinical trials, which explains the scarcity of literature pertaining to their management.

- Each case of synchronous dual malignancies has to be evaluated with the intent of preserving functional autonomy and maximising probability of cure, for which a single treatment modality is usually not appropriate.

- An advanced radiotherapy technique like Image Guided Volumetric Modulated Arc Therapy (IG-VMAT) is well suited for unconventional cases, ensuring better dose distribution, rapid treatment delivery and improved accuracy when compared with intensity modulated radiotherapy.

$9 \mathrm{~mm}$ ), with microscopically involved margin (R1 resection) and no involvement of the mandibular bone, lymphovascular invasion, perineural invasion or nodal metastases (0/72 nodes). The final staging was $\mathrm{pT}_{3} \mathrm{pN}_{0} \mathrm{cM}_{0}$ (AJCC stage III). After an uneventful recovery, he was planned for adjuvant radiotherapy to tongue (based on the risk posed by depth of invasion, $\mathrm{pT}_{3}$ descriptor and R1 resection). Along with delivering adjuvant radiotherapy to tongue, he was also planned for definitive radiotherapy to PFS SqCC. ${ }^{2}$ Radiotherapy planning was challenging as the patient's spine deformity precluded standard immobilisation(figure 2C,D) and target delineation was complicated as the unique position meant that delineation guidelines had to be adapted with close collaboration with a diagnostic radiologist (figure 3). Radiotherapy planning and treatment delivery (via Image Guided Volumetric Modulated Arc Therapy) was performed on Varian Eclipse v13.5 and Varian TrueBeam v2.5 (Varian Medical Systems, Palo Alto), respectively.

The patient tolerated treatment well, with Radiation Therapy Oncology Group grade 2 acute mucositis and xerostomia. Two months after treatment completion, the patient achieved a complete radiological response (figure 4) in the PFS primary and has remained recurrence free for 1 year, with no late side effects.

Contributors IA is the treating junior consultant (radiotherapy), author of the paper, responsible for drafting the manuscript and revising it. He is the guarantor. $\mathrm{RL}$ is the associate consultant (diagnostic radiology), responsible for drafting the imaging-related portions of the manuscript. He also assisted in radiotherapy treatment planning. KSC is the supervising treating consultant (radiotherapy) and participated in article formulation, editing and oversight. SR is the supervising treating consultant (surgical oncology) and participated in article formulation, editing and oversight.

Funding The authors have not declared a specific grant for this research from any funding agency in the public, commercial or not-for-profit sectors.

Competing interests None declared.

Patient consent Obtained.

Provenance and peer review Not commissioned; externally peer reviewed.

(C) BMJ Publishing Group Ltd (unless otherwise stated in the text of the article) 2018. All rights reserved. No commercial use is permitted unless otherwise expressly granted.

\section{REFERENCES}

1 Manon RR, Myers JN, Skinner HD. Oral cavity. In: Halperin EC, Brady LW, Wazer DE, Perez CA, eds. Perez \& Brady's principles and practice of radiation oncology. 6 th ed. Philadelphia: Lippincott Williams \& Wilkins, 2013:795-816.

2 Kruser TJ, Shah HK, Hoffman HT et al. Hypopharynx. In: Halperin EC, Brady LW, Wazer DE, Perez CA, eds. Perez \& Brady's principles and practice of radiation oncology. 6th ed. Philadelphia: Lippincott Williams \& Wilkins, 2013:834-50.

Copyright 2018 BMJ Publishing Group. All rights reserved. For permission to reuse any of this content visit http://group.bmj.com/group/rights-licensing/permissions.

BMJ Case Report Fellows may re-use this article for personal use and teaching without any further permission.

Become a Fellow of BMJ Case Reports today and you can:

- Submit as many cases as you like

- Enjoy fast sympathetic peer review and rapid publication of accepted articles

- Access all the published articles

- Re-use any of the published material for personal use and teaching without further permission

For information on Institutional Fellowships contact consortiasales@bmjgroup.com

Visit casereports.bmj.com for more articles like this and to become a Fellow 